\title{
Applications of the Eigenmodes of the Staggered Dirac Operator
}

\author{
L. Venkataraman and G. Kilcup ${ }^{\mathrm{a}}$

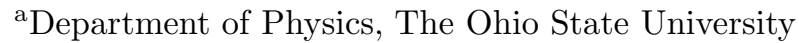 \\ 174 West 18th Ave, Columbus, $\mathrm{OH} 43210$
}

\begin{abstract}
We have constructed the lowest few eigenvectors of the staggered Dirac operator on $S U(3)$ gauge configurations, both quenched and dynamical. We use these modes to study the topological charge and to construct approximate hadronic correlators.
\end{abstract}

\section{Introduction}

In a recent study [1] we successfully calculated the mass of the $\eta^{\prime}$ meson using staggered fermions. According to conventional lore, the $\eta^{\prime}$ receives a large portion of its mass from instanton effects. At the fermionic level, this would mean receiving contributions from zero modes of the Dirac operator. These zero modes come with a definite chirality in the continuum and are associated with the topological charge $(Q)$ of a gauge configuration via the index theorem:

$Q=n_{+}-n_{-}$

where $n_{+}$and $n_{-}$are respectively the number of right and left-handed zero modes. At finite lattice spacing, $\gamma_{5}$ and $\not D$ do not exactly anti-commute, so there are no exact chiral zero modes and nor is the topological charge of gauge configurations well defined. Nevertheless, one might expect to find a few low lying eigenmodes carrying chiral charge. Smit and Vink [3] verified this sort of behavior for $U(1)$ gauge theory in 2 dimensions.

In this work, we set out to study the extent to which the lattice "zero modes" reproduce the continuum picture for QCD. Accordingly, we have constructed the lowest few eigenmodes of staggered $\not D$ on both quenched and dynamical $S U(3)$ gauge configurations (83 samples each) of size $16^{3} \times 32$ corresponding to a lattice spacing of $0.1 \mathrm{fm}$. We have used the subspace iterations method [2] to compute the eigenmodes. The eigenvalues that we obtain have relative errors in the range $10^{-7}$ for the smallest through $10^{-4}$ for the highest.

\section{Topological charge}

The lattice analog of the $U(1)$ axial anomaly equation gives

$Q=m \operatorname{tr}\left(\Gamma_{5} G(x, y)\right) \equiv m \sum_{\lambda} \frac{u_{\lambda}^{\dagger}(x) \Gamma_{5} u_{\lambda}(y)}{i \lambda_{r}+m}$

where $G(x, y)$ is the fermion propagator. The mode by mode contribution to $u_{\lambda}^{\dagger} \Gamma_{5} u_{\lambda}$ on all the dynamical configurations is shown in Figure 1. It appears to diminish as $\lambda$ increases which can be verified quantitatively by calculating the width, shown in the bottom part of figure 1 . This behavior is consistent with the continuum where $u_{\lambda}^{\dagger} \Gamma_{5} u_{\lambda}=0$ for all the non zero eigenvalues. Figure 2 shows a similar set of plots for the quenched ensemble. Although both $\left\langle\Gamma_{5}\right\rangle$ and its width show qualitatively similar behavior as before, they seem to drop more slowly with increasing $\lambda$. The solid line present in all the figures shown so far represents the minimum value of the 32 nd eigenvalue in the ensemble, ie. the line below which we are sure to have found $100 \%$ of the modes. The sum in eqn(2) for $r=2,4,8,24$ and 32 modes at $m a=0.01$ on a typical dynamical configuration is shown in figure 3. The plateau in $Q$ as the number of modes increases implies that the contribution of the higher modes to the sum in eqn 2 is becoming negligibly small. Although, the plot shown here is for one configuration, a similar behavior is seen on all the configurations, quenched and dynamical. In Figure 3, the isolated point represented by a square is the value of $Q$ obtained from pseudofermions. The "true" value obtained by averaging over 82 

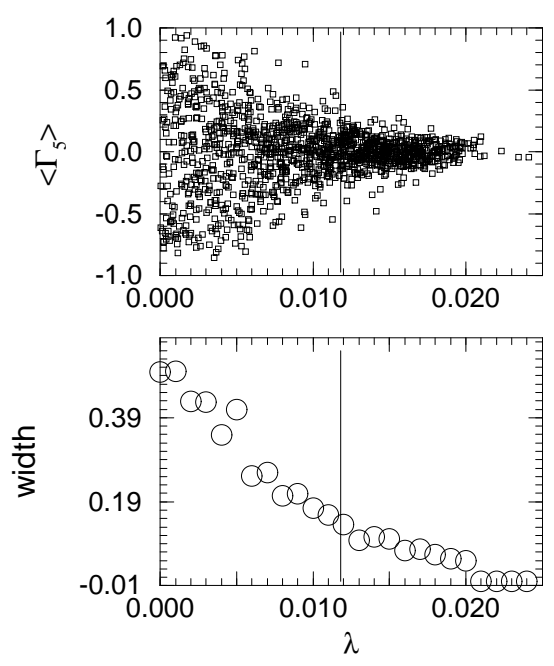

Figure 1. Upper: $\left\langle\Gamma_{5}\right\rangle$ on the dynamical ensemble. Lower: Fluctuations in $\left\langle\Gamma_{5}\right\rangle$.

copies of noise lies within the band marked by the dashed lines. Since the plateau in $Q$ lies within this band it is a very good indication that these lowest modes are the topological zero modes which make the dominant contribution to the sum in eqn 2. We have compared $Q$ obtained from pseudofermions and modes on all the dynamical configurations and find that both agree within errors. On the quenched ensemble, the difference between the two estimators appears to be statistically significant. This simply reinforces the inference reached by comparing Figures 11 and 2 that more than 32 lowest eigenvalues are required to identify an upper limit for the number of shifted zero modes on the quenched configurations.

\section{Hadron Correlators}

Calculation of hadron correlators on the lattice normally involves inverting $\not D+m$ to obtain the quark propagator. When resolved in terms of the eigenvalues and eigenvectors of $\not D$, on a fixed background gauge field, it takes the form

$G(x, y)=\sum_{\lambda} \frac{u_{\lambda}(x) u_{\lambda}^{\dagger}(y)}{i \lambda+m}$.
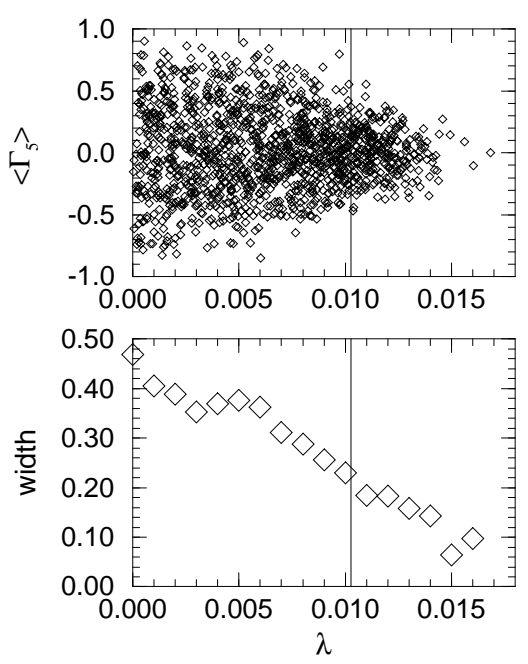

Figure 2. Above: $\left\langle\Gamma_{5}\right\rangle$ on the quenched ensemble. Below:Fluctuations in $\left\langle\Gamma_{5}\right\rangle$.

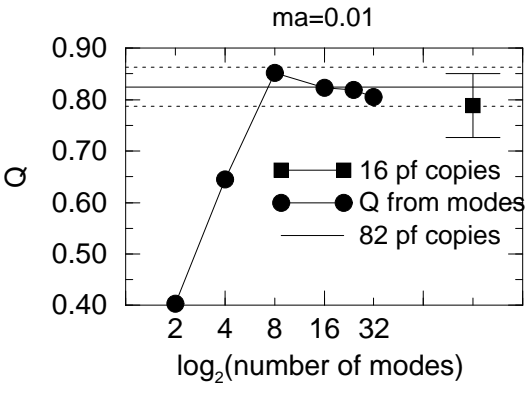

Figure 3. Topological charge on a typical dynamical configuration

Comparing the full propagator obtained using standard inversion procedures like CG, with that constructed out of the sum above with the available eigenmodes gives an insight into the role of the eigenmodes in determining hadronic quantities.

The $\eta^{\prime}$ meson, being a flavor singlet, has both connected and disconnected contributions to its correlator. The discussion in section 2 suggests an extension of the analysis to the $\eta^{\prime}$ disconnected correlator which is given by $\left\langle\operatorname{Tr}\left(\Gamma_{5} G(x, x)\right) \operatorname{Tr}\left(\Gamma_{5} G(y, y)\right)\right\rangle$. We calculate this quantity using pseudofermions and with the available 32 modes. The ratio $R(t)$ of disconnected to the connected correlator for $m a=0.01$ is shown in fig 1 . For the connected correlator, we use the 
full result available from our previous study [1]. On the dynamical configurations, $R(t)$ is fit to the form [1] $R(t)=\frac{N_{v}}{N_{f}}\left[1-\exp \left(-\left(m_{\eta^{\prime}}-m_{8}\right) t\right)\right]$. For staggered fermions, $N_{v}$, the number of valence fermions equals 4 while $m_{8}^{2} \equiv\left(4 m_{K}^{2}+3 m_{\pi}^{2}+\right.$ $\left.m_{\eta}^{2}\right) / 8$. It is evident from the figure that $R(t)$ data with 32 modes agree within error with the full result. We find similarly good agreement

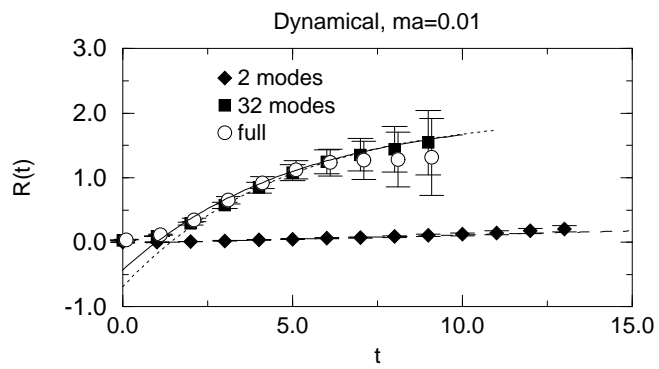

Figure 4. Pion- $\eta^{\prime}$ splitting.

with only 24 modes though 16 appears to be not quite enough. The pattern is the same at the heavier quark masses, 0.02 and 0.03 .

We note that this behavior is not automatic as 32 modes are insufficient to yield a good approximation to the true quark propagator. We find, for example, that the pion is off by a large factor. Based on our knowledge of the staggered eigenvalue spectrum and on recent work done by Negele et al. [4] with Wilson fermions, one might expect that 100-200 modes would be needed to reproduce the pion propagator. Thus the $\eta^{\prime}$ is special in exhibiting an extraordinary sensitivity to the lowest few modes.

The quark propagator, constructed out of the available 32 modes, can be used as a starting seed for calculating $(\not D+m)^{-1}$ using CG. The benefit of using such approximate solutions as a starting iterate in the CG procedure is illustrated in Figure 5 . The number of CG iterations required for the residual to converge to a given tolerance drops by more than $50 \%$ at $m a=0.01$. At higher quark masses, this effect is not so pronounced owing to the fact that the lowest 32 eigenvalues are smaller than the quark mass itself. These conclusions hold only for delta function sources or gauge fixed wall sources built out of delta functions. Quark

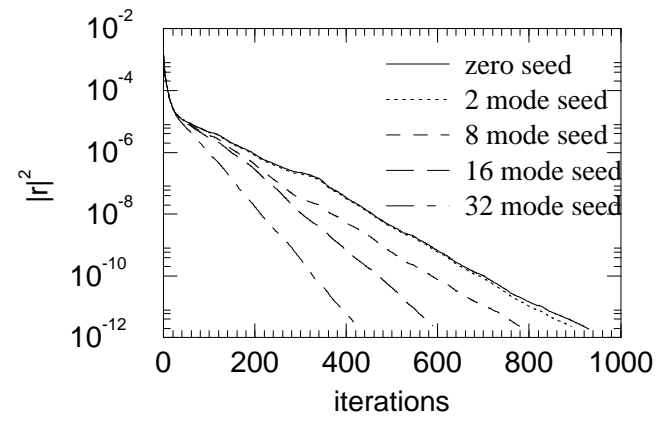

Figure 5. Comparison of CG convergence.

propagators from noisy sources are dominated by short distance modes and hence there is virtually no improvement in the CG convergence rate when the starting seed is built out of long wavelength modes.

\section{Conclusions}

We find that it is possible to identify the shifted zero modes on the lattice since they make the dominant contribution to $u_{\lambda} \Gamma_{5} u_{\lambda}$ and $\operatorname{tr}\left(\Gamma_{5} G(x, y)\right)$. On our dynamical configurations, it seems that the number of topological zero modes must be less than 32 while on the quenched configurations the data indicates that there are more than this number. For most hadronic quantities, the approximate propagator constructed out of these topological zero modes is not accurate enough to be useful. However, a remarkable result of our study is that we find that the $\eta^{\prime}$ flavor singlet interaction on the dynamical configurations is dominated entirely by the quasi-zero modes of $\not D$.

\section{REFERENCES}

1. L. Venkataraman, G. Kilcup and J. Grandy, Nucl. Phys. B53 Proc. Suppl. (1997) 259.

2. B. Bunk, Nucl. Phys. B53 Proc. Suppl. (1997) 987.

3. J. Smit and J. C. Vink, Nucl. Phys. B286 (1987) 485.

4. T. L. Ivanenko and J. W. Negele, this proceedings. 